\title{
Attentional scanning and space errors
}

\author{
SERGIO CESARE MASIN \\ University of Padua, Padua, Italy \\ and \\ ANNA AGOSTINI \\ University of Udine, Udine, Italy
}

\begin{abstract}
Using the method of paired comparisons, pairs of simultaneous horizontal or vertical lines, with one line above and one below or one on the left and one on the right of a fixation point, respectively, were presented tachistoscopically for length comparison. Space errors were found to have a pattern similar to that of time errors. The tendency to guess the comparative response from the absolute magnitude of stimuli is proposed as a basis for time and space errors. Manipulation of attentional scanning, which implies a more frequent usage of this guessing strategy for one of the two lines in a pair, was shown to affect space errors.
\end{abstract}

Using the method of paired comparisons, we investigated the possible role of attentional scanning in the comparative judgment of brief, simultaneous stimuli. First, let us consider an overview of this method and the judgment errors inherent in it.

The method of paired comparisons uses two sets of variable stimuli, with each set the identical copy of the other. These variables vary with respect to a single attribute. Let $A_{i}(i=1,2, \ldots, n)$ and $B_{j}(j=1,2, \ldots, n)$ denote the variables in the two sets, in which $i$ and $j$ increase with the varied attribute. Each $\boldsymbol{A}_{i}$ is paired with each $\boldsymbol{B}_{j}$. For each ensuing pair, subjects usually report whether $A_{i}<B_{j}$ or $A_{i}>B_{j}$.

Each $A_{i}$ or $B_{j}$ may be taken as a variable or standard. When $A_{i}$ is the standard, the variables are $B_{1}$ to $B_{n}$. When $B_{j}$ is the standard, the variables are $A_{1}$ to $A_{n}$. Each standard involves one point of subjective equality (PSE), which is the physical value of the varied attribute that has a $50 \%$ probability of being reported as larger than the physical value of the standard. Let $\mathrm{PSE}_{i}$ and $\mathrm{PSE}_{j}$ be the PSEs for $A_{i}$ and $B_{j}$, respectively. The differences $\mathrm{PSE}_{i}-A_{i}$ and $\mathrm{PSE}_{j}-B_{j}$ are called either time errors or space errors when the stimuli in a pair are either successive or simultaneous, respectively (Guilford, 1954).

When $A_{i}$ and $B_{j}$ are successive and $A_{i}$ follows $B_{j}$, the $\mathrm{PSE}_{i}$ tends to be larger than the $\mathrm{PSE}_{j}$, for large standards, and smaller than the $\mathrm{PSE}_{j}$, for small standards. That is, with PSE as the dependent variable, there is an interaction between the factors value of $A_{i}$, or $B_{j}$, and (temporal) order of $A_{i}$ and $B_{j}$ in a pair (Masin \& Agostini, 1990). Here, this interaction is called the value-order interaction.

This research was supported by a grant from CNR (the Italian $\mathrm{Na}$ tional Research Council), and part of it was presented at the 31st Annual Meeting of the Psychonomic Society, New Orleans, November 1990. Correspondence should be addressed to S. C. Masin at the University of Padua, Department of General Psychology, Piazza Capitaniato 3, 35100 Padova, Italy.
When they are compared, two successive stimuli seem to receive a code proportional to the absolute magnitude of the comparison attribute. The two codes are then matched to produce the comparative response (Bower, 1971). The retrieval of the first stimulus code for matching with the second requires time. In addition, this retrieval may occasionally fail. Consequently, the comparative response may occasionally be produced on the basis of only the second stimulus code. It has been suggested that when the second stimulus code is "large" ("small") and the first stimulus code is not used, the "larger" ("smaller") comparative response tends to be guessed (Masin, 1988; Masin \& Fanton, 1989). As we shall see, this bias implies the occurrence of the value-order interaction.

Let us now consider the comparative judgment of two simultaneous tachistoscopic stimuli, one on the left and one on the right of a fixation point. Horizontal letter arrays presented tachistoscopically tend to be scanned from left to right (White, 1969). We hypothesize that the task of comparing two horizontal, simultaneous visual stimuli activates this attentional scanning tendency. That is, at the onset of two horizontal, simultaneous stimuli presented for comparison, there is a greater probability that the left stimulus would be attended to first. Consequently, left tachistoscopic stimuli would be encoded at a higher rate more often than right stimuli (Downing, 1988; LaBerge \& Brown, 1989; Reinitz, 1990), and premature termination of encoding would be more frequent for right than for left stimuli (Klein \& Farrell, 1989). It follows that for short stimulus durations, the comparative response would be produced, at least occasionally, on the basis of only the left stimulus code.

As we did for time errors, we suggest that when the left stimulus code is large (small) and the right stimulus code is not used, the larger (smaller) comparative response tends to be guessed. Thus, it may be predicted that the value-order interaction occurs for both time and space 
errors, with the left stimulus in a pair of simultaneous stimuli equivalent to the last stimulus in a pair of successive stimuli. The following experiment tested this prediction.

\section{EXPERIMENT 1}

\section{Method}

Subjects. Eighty students from the Faculty of Foreign Languages and Literature at the University of Udine participated. Their reported vision was either normal or corrected to normal by lenses.

Stimuli. Stimuli were pairs of black vertical lines displayed for $125 \mathrm{msec}$ on a light-gray $22 \times 30 \mathrm{~cm}$ IBM 8514 color-display screen controlled by an IBM 80 computer. These pairs were all combinations of five lines that varied in $0.12-\mathrm{cm}$ steps, the shortest being $8.38 \mathrm{~cm}$. Line width was 1 pixel. Viewing distance was $65 \mathrm{~cm}$. The lines in a pair were placed symmetrically at $8 \mathrm{~cm}\left(7^{\circ}\right)$ from the fixation point, 1 yellow pixel always present in the middle of the screen. This pixel and the centers of the two lines lay on an imaginary horizontal line. Each stimulus was signaled $1.4 \mathrm{sec}$ before its appearance by a very brief sound. Each pair of lines appeared $2.7 \mathrm{sec}$ after a response was typed.

The experimental room was lighted at a constant, normal illumination level by neon tubes on the ceiling. The subjects viewed the stimuli through an $8.5 \times 14.5 \mathrm{~cm}$ rectangular cutout in a black 70 $\times 100 \mathrm{~cm}$ cardboard placed $49 \mathrm{~cm}$ from the screen. Eye level was adjusted by a chin-and-head-rest.

Procedure. The method of paired comparisons was used. Let the variables on the left and the right of the fixation point be $A_{i}$ and $B_{j}$, respectively, with the integers $i$ and $j$ increasing from 1 to 5 with length. Each $A_{i}$ was paired with each $B_{j}$. To each subject, the ensuing $25 A_{i}, B_{j}$ pairs were shown randomly twice consecutively, that is, one set of 25 after the other set, in a single session with no rest period. The subjects did not know that lines were to be named " $A$ " or " $B$."

The subjects were asked to focus on the fixation point at the acoustic signal. It was explained to them that avoiding eye movements during fixation would optimize performance and permit equal visibility of the lines in a pair. The subjects were asked to verbally identify the longer line in a pair. The "equal" or "don't know" responses were forbidden. Each subject gave 50 responses in total.

\section{Results}

For $i=j, A_{i}=B_{j}$. Consequently, for each $i=j$, two PSEs derive from a single application of the method of

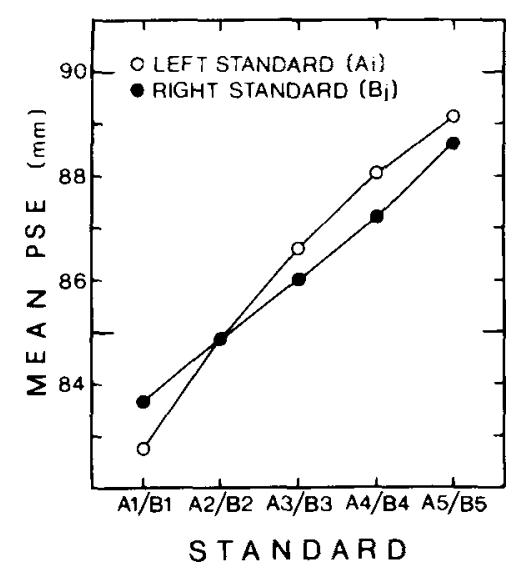

Figure 1. Mean point of subjective equality (PSE) as a function of left $\left(A_{i}\right)$ or right $\left(B_{j}\right)$ standards. paired comparisons-one when $A_{i}$ is taken as the standard and one when $B_{j}$ is taken as the standard.

Figure 1 shows the mean PSEs as a function of size of standard, for left ( $A_{i}$, open circles) and right standards $\left(B_{j}\right.$, filled circles). The mean PSEs are means of 160 individual PSEs (two replicates) computed by the simplified version of the method of transitions (Masin, 1989; Masin \& Vidotto, 1984); they correspond substantially to those obtained by the weighted least-squares procedure. A 2 (spatial order of stimuli in a pair) $\times 5$ (value of standard) analysis of variance (ANOVA) with two replicates showed that the main effects caused by spatial order were significant $[F(1,79)=5.1, p<.05]$, indicating an overestimation of left lines (Brown, 1953; Ogle, 1950; Takala, 1951). The value-order interaction was significant $[F(4,316)$ $=92.8, p<.0001]$, which suggests that time and space errors might have a common basis.

\section{Discussion}

The value-order interaction in Figure 1 could be explained as follows. For simplicity, let us consider only the extreme values of $A_{i}$ and $B_{j}$. When $A_{1}$ is compared with the shortest variable $\left[B_{1}\left(=A_{1}\right)\right]$, the observed probability to respond "shorter" to $A_{1}$ is greater than the expected or theoretical probability $(P)$, because $A_{1}$ has a high probability to be coded as "short" and is more likely to be attended to first. Therefore, the proportion of "longer" responses to $B_{1}$ tends to be higher than $P$. This higher proportion implies a shift of the psychometric function (and $\mathrm{PSE}_{1}$ ) away from the middle of the range of variables. When $A_{5}$ is compared with the longest variable $\left[B_{5}\right.$ $\left(=A_{5}\right)$ ], the probability to respond "longer" to $A_{3}$ is greater than $P$, because $A_{5}$ has a high probability to be coded as "long" and is more likely to be attended to first. The proportion of "longer" responses to $B_{5}$ tends, then, to be lower than $P$, again implying a shift of the psychometric function (and PSE s $_{5}$ away from the middle of the range of variables. Analogous reasonings show that PSE $_{1}$ and PSE $_{5}$ shift toward the middle of the range of variables when $B_{1}$ or $B_{5}$ are right standards. This interpretation is strengthened by the finding that the left line in a pair has greater weight on the comparative response (Hellström, 1990).

However, it is also possible that the value-order interaction in Figure 1 depends on a difference in cerebral hemisphere specialization for spatial judgment. ${ }^{1}$ In fact, Kosslyn et al. (1989; see also Kosslyn, 1987) found faster finger reaction times in evaluations of visual distance when stimuli were initially presented in the left visual hemifield. ${ }^{2}$ To examine this possibility, Experiment 1 was repeated with the simultaneous lines in a pair now placed above and below the fixation point.

\section{EXPERIMENT 2}

\section{Method}

Subjects. Twenty different students, recruited according to the criteria used in Experiment 1, participated.

Stimuli. Stimuli and stimulus conditions were the same as in Experiment 1 , except for the following changes. The lines varied in 


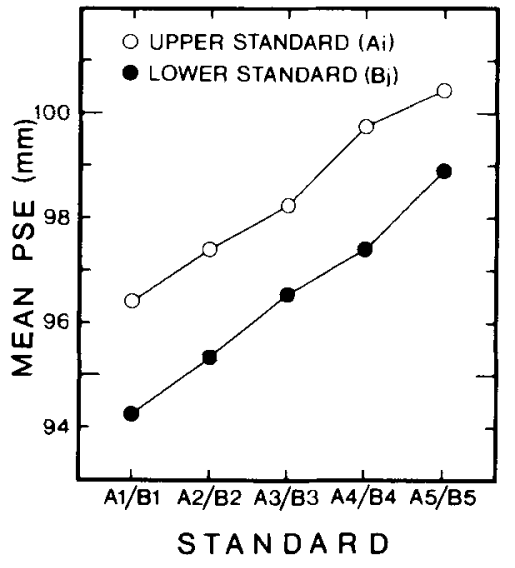

Figure 2. Mean point of subjective equality (PSE) as a function of upper $\left(A_{i}\right)$ or lower $\left(B_{j}\right)$ standards.

$0.09-\mathrm{cm}$ steps, the shortest being $9.57 \mathrm{~cm}$. The lines in a pair were horizontal and placed symmetrically at $4 \mathrm{~cm}\left(3.5^{\circ}\right)$ from the fixation point. The centers of the two lines and the fixation point lay on an imaginary vertical line.

Procedure. Let the upper and lower lines in a pair be $A_{i}$ and $B_{j}$, respectively. The procedure was the same as it was in Experiment 1, except that now the $25 A_{i}, B_{j}$ pairs were presented six times consecutively, with a 1-2 min rest period between the second and third and between the fourth and fifth sets of presentations.

\section{Results}

Figure 2 shows the mean PSEs as a function of size of standard, for upper ( $A_{i}$, open circles) and lower standards $\left(B_{j}\right.$, filled circles). The mean PSEs are means of 120 individual PSEs (six replicates) computed as they were in Experiment 1. A 2 (spatial order) $\times 5$ (value of standard) ANOVA with six replicates showed that the main effects caused by spatial order were significant $[F(1,19)=320$, $p<.0001]$, in agreement with the well-known overestimation of the upper half of visual stimuli in normal viewing conditions (Robinson, 1972, p. 104). The value-order interaction was significant $[F(4,76)=22.0, p<.0001]$.

\section{Discussion}

On the basis of the present data, the possibility that a hemispheric asymmetry for spatial judgment contributes to space errors cannot be excluded. However, the occurrence of the value-order interaction with lines in the upper and lower visual hemifields indicates that some factor other than cerebral hemifield specialization is operating. We suggest that this other factor is attentional scanning. That is, the value-order interaction in Figure 2 would be the result of a slight tendency to start the comparison from the lower line in a pair.

If attentional scanning is a basis of space errors, then a manipulation of this scanning should affect the value-order interaction. Accordingly, Experiments 3 and 4 tested the hypothesis that space errors are affected when the probability of attending first to one line in a pair is varied. In Experiments 3 and 4, the probability of focusing attention first on one line in a pair was maximized by having the subjects rate the length of that line before producing the comparative response.

\section{EXPERIMENT 3}

\section{Method}

Subjects. Subjects were 96 different university students recruited according to the criteria used in Experiment 1. They were divided into two groups of 48 ( $X$ and $Y$ ).

Stimuli. Stimuli were the same as in Experiment 1.

Procedure. The method of paired comparisons was used. The $25 A_{i}, B_{j}$ pairs were presented randomly four times consecutively in a single session, with a 1-2 min rest period between the second and the third sets of presentations. As in Experiment 1, the subjects were carefully instructed to focus correctly on the fixation point. For each pair, subjects were asked to rate the length of one line as soon as possible and, immediately after the rating, to compare the length of the rated line with that of the other line in the same pair. Initially, the two groups were shown the five lines (half of each group of subjects in ascending order and half in descending order) in the middle of the screen, one every $1 \mathrm{sec}$. The integers from 1 to 9 were used for the rating, with 1 corresponding to the shortest line and 9 to the longest line. For each pair, Group X (Y) rated only left (right) lines in the first two sets of presentations, and only right (left) lines in the second two. The instructions for the comparative response were the same as they were in Experiment 1 . Each subject produced 100 absolute and 100 comparative responses.

\section{Results}

Figure 3 shows the mean PSEs as a function of $A_{i}$ and $B_{j}$ taken as left and right standards, respectively. These mean PSEs are means of 192 individual PSEs (two replicates) computed as they were in Experiment 1. Open squares (filled circles) show the results when left (right) lines were rated.

A 2 (spatial order) $\times 2$ (direction of scanning) $\times 5$ (value of standard) ANOVA with two replicates showed that the main effects caused by spatial order and direction of scanning were statistically significant $[F(1,95)=$ $306, p<.0001$, and $F(1,95)=7.67, p<.01$, respectively]. These results confirm that left lines are overestimated and indicate that deployment of attention on left lines contributes slightly to this overestimation (Coren \&

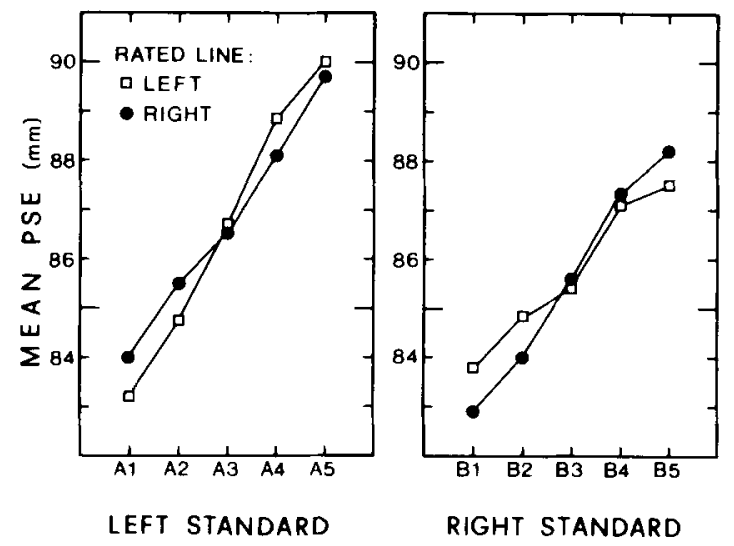

Figure 3. Mean point of subjective equality (PSE) as a function of left $\left(A_{i}\right)$ and right $\left(B_{j}\right)$ standards, for left and right rated lines. 
Girgus, 1972; Fraisse, Ehrlich, \& Vurpillot, 1956; Jordan \& English, 1989; Reuter-Lorenz, Kinsbourne, \& Moscovitch, 1990). ${ }^{3}$ The significant interactions between spatial order and direction of scanning $[F(1,95)=4.33$, $p<.05]$, spatial order and value of standard $[F(4,380)$ $=235, p<.0001]$, direction of scanning and value of standard $[F(4,380)=11.5, p<.0001]$, and spatial order, direction of scanning, and value of standard $[F(4,380)=237, p<.0001]$, confirm the occurrence of the value-order interaction found in Experiment 1 and show that space errors vary with the probability of focusing attention first on one line in a pair. Thus, the value-order interaction is affected when attentional scanning is manipulated.

\section{EXPERIMENT 4}

Experiment 4 was a repetition of Experiment 3, with the simultaneous lines in a pair now placed above and below the fixation point.

\section{Method}

Subjects. Subjects were 24 university students from the Department of General Psychology at the University of Padua. Their reported vision was either normal or corrected to normal by lenses.

Stimuli. Stimuli were the same as in Experiment 2, except for the following changes. Lines were displayed for $30 \mathrm{msec}$ on a 22 $\times 35 \mathrm{~cm}$ light-gray background on an EIZO 9400 color-display screen controlled by an ASEM 9030 computer. The lines varied in $0.11-\mathrm{cm}$ steps, the shortest being $11.84 \mathrm{~cm}$. The distance of a line from the fixation point was $6.2 \mathrm{~cm}\left(6.5^{\circ}\right)$. Viewing distance was $55 \mathrm{~cm}$.

Procedure. The procedure was the same as in Experiment 3. Groups $\mathrm{X}$ and $\mathrm{Y}$ were now made up of 12 subjects each.

\section{Results}

Figure 4 shows the mean PSEs as a function of the upper $\left(A_{i}\right)$ and lower $\left(B_{j}\right)$ lines in a pair taken as standards.

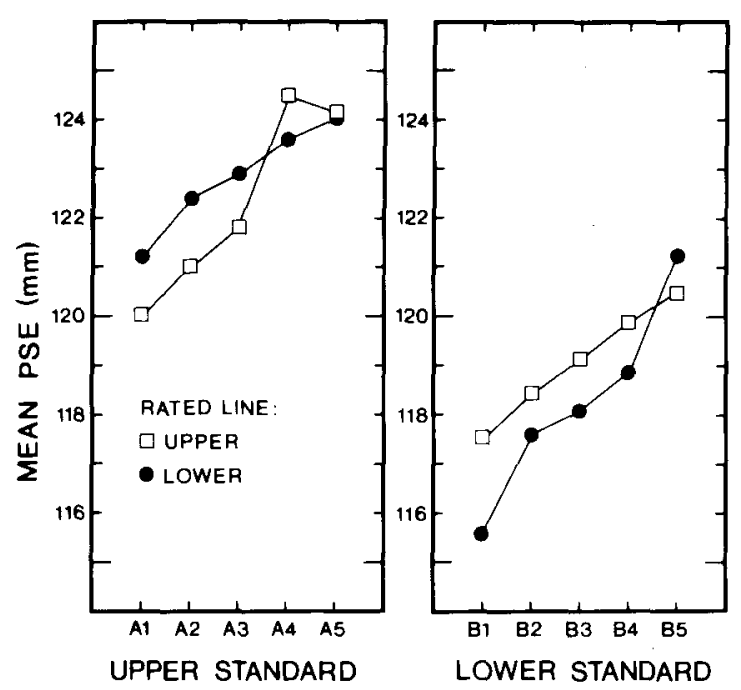

Figure 4. Mean point of subjective equality (PSE) as a function of upper $\left(A_{i}\right)$ and lower $\left(B_{j}\right)$ standards, for upper and lower rated lines.
The mean PSEs are means of 48 individual PSEs (two replicates) computed as they were in Experiment 1. Open squares (filled circles) show the results when upper (lower) lines were rated.

A 2 (spatial order) $\times 2$ (direction of scanning) $\times 5$ (value of standard) ANOVA with two replicates showed that the main effects due to spatial order and direction of scanning were statistically significant $[F(1,23)=1,672$, $p<.0001$, and $F(1,23)=35.4, p<.0001$, respectively]. The results confirm that upper lines are overestimated and indicate that attention to upper lines contributes slightly to this overestimation. ${ }^{4}$ The significant interactions between spatial order and direction of scanning $[F(1,23)=61.5, p<.0001]$, spatial order and value of standard $[F(4,92)=43.0, p<.0001]$, direction of scanning and value of standard $[F(4,92)=35.0$, $p<.00011$, and spatial order, direction of scanning, and value of standard $[F(4,92)=73.3, p<.0001]$ confirm that the value-order interaction changes markedly when attentional scanning is induced artificially by focusing the subject's attention first on one line in a pair. These results seem difficult to interpret in terms of a hemispheric asymmetry for spatial judgment.

\section{CONCLUSION}

Without excluding the possibility that space errors may also depend on a hemispheric asymmetry for spatial judgment, the present results favor the following conclusion: Time and space errors seem to have one common basisthe tendency to guess the comparative response from only one of the compared stimuli when the code of the other stimulus cannot be used. In a set of stimuli, large (small) stimuli have a high probability of being larger (smaller) than another unknown stimulus from the same set. It is plausible that subjects intuitively use this knowledge to guess the comparative response.

\section{REFERENCES}

Bower, G. (1971). Adaptation-level coding of stimuli and serial position effects. In M. H. Appley (Ed.), Adaptation-level theory (pp. 175201). New York: Academic Press.

Brown, K. T. (1953). Factors affecting differences in apparent size between opposite halves of a visual meridian. Journal of the Optical Society of America, 43, 464-472.

Coren, S., \& Girgus, J. S. (1972). Differentiation and decrement in the Müller-Lyer illusion. Perception \& Psychophysics, 12, 466-470. DownING, C. J. (1988). Expectancy and visual-spatial attention: Effects on perceptual quality. Joumal of Experimental Psychology: Human Perception \& Performance, 14, 188-202.

Fraisse, P., Ehrlich, S., \& Vurpillot, E. (1956). Études de la centration perceptive par la méthode tachistoscopique. Archives de Psychologie, 35, 193-214.

GuILfoRd, J. P. (1954). Psychometric methods. New York: McGraw-Hill.

HAMPSON, E., KIMURA, D. (1984). Hand movement asymmetries during verbal and nonverbal tasks. Canadian Joumal of Psychology, 38, 102-125.

Helsström, A. (1990). Neuropsychological applications of stimulus comparison tasks. In F. Müller (Ed.), Fechner Day 90. Proceedings of the Sixth Annual Meeting of the International Society for Psychophysics (pp. 205-209). Würzburg, Germany: International Society for Psychophysics. 
Jordan, K., English, P. W. (1989). Simultaneous sampling and length contrast. Perception \& Psychophysics, 46, 546-554.

KleIN, R., \& FarRell, M. (1989). Search performance without eye movements. Perception \& Psychophysics, 46, 476-482.

KossLyn, S. M. (1987). Seeing and imagining in the cerebral hemispheres. A computational approach. Psychological Review, 94, 148-175.

Kosslyn, S. M., Koenig, O., Barrett, A., Cave, C. B., Tang, J., \& Gabrieli, J. D. E. (1989). Evidence for two types of spatial representations: Hemispheric specialization for categorical and coordinate relations. Journal of Experimental Psychology: Human Perception \& Performance, 15, 723-735.

LABerge, D., \& Brown, V. (1989). Theory of attentional operations in shape identification. Psychological Review, 96, 101-124.

MASIN, S. C. (1988, November). New data and an explanation for the time- and space-errors. Paper presented at the 29th Annual Meeting of the Psychonomic Society, Chicago.

MASIN, S. C. (1989). A simplified version of the method of transitions. Perceptual \& Motor Skills, 68, 131-135.

Masin, S. C., AGostini, A. (1990). Time errors in the method of pair comparisons. American Journal of Psychology, 103, 487-494.

MAsin, S. C., \& AGostini, A. (1991). Handedness and space errors. Bulletin of the Psychonomic Society, 29, 301-303.

MAsIN, S. C., FANTON, V. (1989). An explanation for the presentationorder effect in the method of constant stimuli. Perception \& Psychophysics, 46, 483-486.

Masin, S. C., FAnton, V., Crestoni, L. (1988). An experimental study of the asymmetry effect in the method of constant stimuli. Psychological Research, 50, 181-182.

Masin, S. C., \& Vidotro, G. (1984). The method of transitions. Perception \& Psychophysics, 36, 593-594.

OGLE, K. N. (1950). Researches in binocular vision. Philadelphia: Saunders.

Polich, J., DeFrancesco, D. P., Garon, J. F., \& Cohen, W. (1990). Hemispheric differences in visual search of simple line arrays. Psychological Research, 52, 54-61.

ReINITZ, M. T. (1990). Effects of spatially directed attention on visual encoding. Perception \& Psychophysics, 47, 497-505.

Reuter-Lorenz, P. A., Kinsbourne, M., \& Moscovitch, M. (1990). Hemispherical control of spatial attention. Brain \& Cognition, 12, 240-266.

RoBinson, J. O. (1972). The psychology of visual illusions. London: Hutchinson.
TakalA, M. (1951). Asymmetries in the visual space. Helsinki: Finnish Literature Society Press.

Verfaelde, M., \&eilman, K. M. (1990). Hemispheric asymmetries in attentional control: Implications for hand preference in sensorimotor tasks. Brain \& Cognition, 14, 70-80.

White, M. J. (1969). Laterality differences in perception: A review. Psychological Bulletin, 72, 387-405.

Wünscher, T., Jordan, K., Gille, H-G., Roth, N. (1990). A new approach to cerebral asymmetry: RT differences in simultaneous bimanual finger movement during verbal and nonverbal tasks. Perceptual \& Motor Skills, 70, 543-548.

\section{NOTES}

1. For the suggestion of two concurrent judgmental factors in time errors, see Masin and Agostini (1990, p. 492).

2. Kosslyn et al. (1989) had subjects respond with both hands to amoeba-like figures with a dot on either the contour or the outside of the figure. The interpretation of their results is difficult, because (1) there is evidence of an interaction between overall attributes of visual stimuli and hemispheric-processing differences in visual search (Polich, DeFrancesco, Garon, \& Cohen, 1990), (2) there are complex and partly unknown reciprocal influences of the attentional and intentional systems (Verfaellie \& Heilman, 1990), and (3) judgments of nonverbal visual stimuli involve a shift in hand preference (Hampson \& Kimura, 1984; Wünscher, Jordan, Gille, \& Roth, 1990). In agreement with Points 2 and 3, Masin, Fanton, \& Crestoni (1988) obtained data showing that space errors resulting from a manual response are different from space errors resulting from a verbal response, and Masin \& Agostini (1991) found that the patterns of space errors in left- and right-handed subjects who respond with both hands are specular.

3. Line ratings were asked only as a means to direct attention toward one of the two lines in a pair. The grand mean ratings of left and right line lengths were 6.82 and 6.68 , respectively. The information conveyed by these means is uncertain, because each line rating lacked the corresponding rating of the other line in the same pair for proper comparison.

4. The grand mean ratings of upper and lower line lengths were 6.90 and 6.74 , respectively. See Note 3 .

(Manuscript received November 8, 1990; revision accepted for publication March 21, 1991.) 